Faculdade de Ciências Econômicas UFRGS
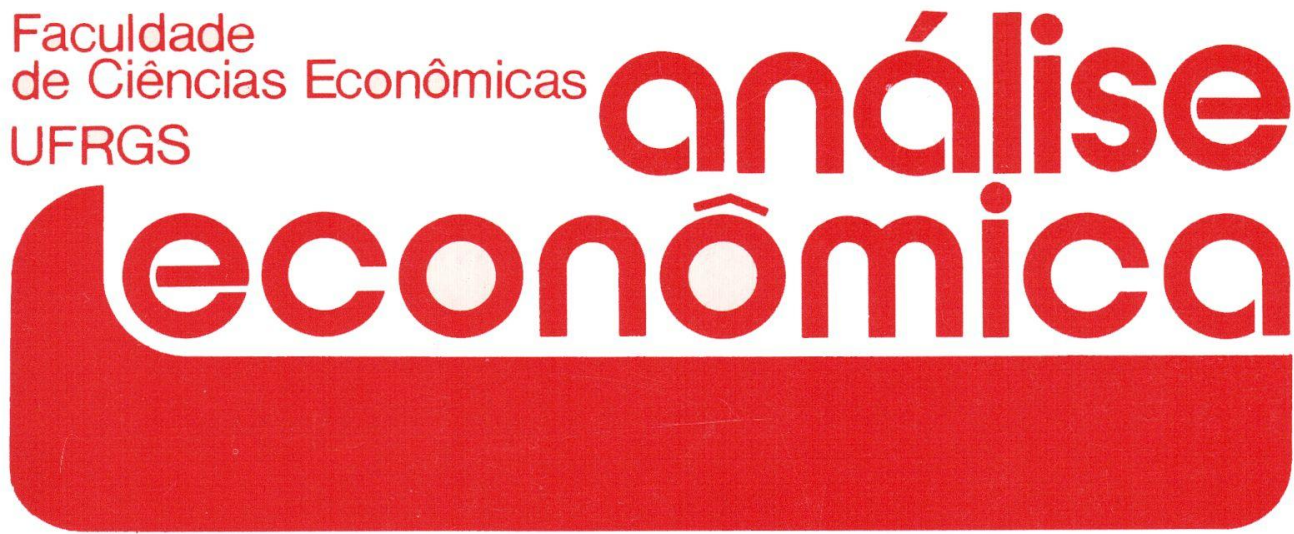

- INDEXAÇĀo SALARIAL: UMA ABORDAGEM MACROECONÓMICA Jo Anna Grav

- AJUSTE NO EMPREGO E PRODUTIVIDADE NA DECADA DE OITENTA

Carlos Antônio Luque José Paulo Zeeteno Chahad

- O CONSUMIDOR KEYNESIANO Marcelo Córtes Neri

- HETEROGENEIDADE DO TRABALHO E TAXA DE LUCRO EM MARX

Francisco Cribari Neto

- ECONOMIAS DE ESCALA: UMA REVISĀO Jesiel de Marco Gomes

- Concentraçấo bancária no BRASIL

Marcelo Resende

- NOYOS RUMOS PARA O SETOR ELETRICO NO BRASIL

Adriano Pires Rodrigues

Eduardo da Cunha Vianna

- OFERTA E DEMANDA DE FRANGO DE CORTE NO BRASIL

Narciso Gonçalves de Castro et alii

- ANÁLISE ECONÔMICA DA IRRIGAÇÃo DO MILHO

Lúcia M Schirmer

Juvir Luiz Mattuella

- REFLORESTAMENTO NO BRASIL. Carlos José Caetano Bacha

- ESCOLHA DE TECNOLOGIA EM ESTRUTURA DE PRINCIPAL AGENTE Kyle D. Kauffma:

- A QUESTÁo DEMOGRÁfica e A PRAXEOLOGIA

Anton Karl Biedermann et alii
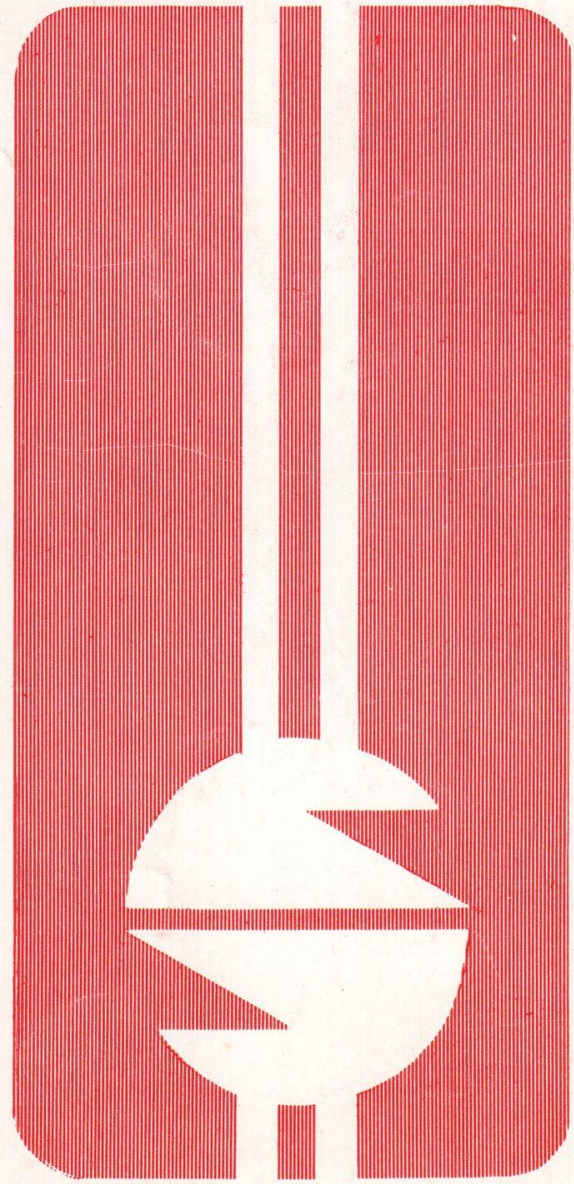
UNIVERSIDADE FEDERAL DO RIO GRANDE DO SUL

Reitor: Prof. Tuiskon Dick

FACULDADE DE CIÉNCIAS ECONÔMICAS

Diretora: Prof ${ }^{\text {a }}$ Yeda Rorato Crusius.

CENTRO DE ESTUDOS E PESQUISAS ECONÔMICAS

Diretor: Reinaldo Ignacio Adams

DEPARTAMENTO DE CIÉNCIAS ECONÔMICAS

Chefe: Prof. Fernando Ferrari Filho

CURSO DE POS-GRADUAÇÄO EM ECONOMIA

Coordenador: Prof. Nali de Jesus de Souza

CURSO DE PÓS-GRADUAÇĀO EM ECONOMIA RURAL

Coordenador: Prof. Atos Freitas Grawunder

CONSELHO EDITORIAL: Achyles Barcelos da Costa, Aray Miguel Feldens, Atos Freitas Grawunder, Carlos Augusto Crusius, Ernani Hickmann, João Rogério Sanson, Juvir Luiz Mattuella, Maria Imilda da Costa e Silva, Nali de Jesus de Souza, Nuno Renan Lopes de Figueiredo Pinto, Otilia Beatriz Kroeff Carrion, Otto Guilherme Konzen, Paulo Alexandre Spohr, Pedro Cezar Dutra Fonseca, Reinaldo Ignacio Adams, Roberto Camps Moraes, Valter José Stülp, Yeda Rorato Crusius, David Garlow (Wharton Econometrics Forecasts Association, E.U.A.), Edgar Augusto Lanzer (UFSC), Eleutério F.S. Prado (USF), Fernando Holanda Barbosa (FGV/RJ), Gustavo Franco (PUC/RJ), Joaquim Pinto de Andrade (UnB), Juan H. Moldau (USP), Werner Baer (Univ. de Illinois, E.U.A.).

COMISSĀO EDITORIAL: Atos Freitas Grawunder, Pedro Cezar Dutra Fonseca, Reinaldo Ignacio Adams e Roberto Camps Moraes.

EDITOR: Nali de Jesus de Souza

SECRETARIA: Maria Ivone de Mello (normalização), Vanete Ricacheski (revisão de textos).

FUNDADOR: Prof. Antônio Carlos Santos Rosa

Os materiais publicados na revista Análise Econômica são de exclusiva responsabilidade dos autores. É permitida a reprodução total ou parcial dos trabalhos, desde que seja citada a fonte.

Aceita-se permuta com revistas congêneres. Aceitam-se, também, livros para divulgação, elaboração de resenhas ou recenșões.

Toda correspondência, material para publicação, assinaturas e - permutas devem ser dirigidos ao seguinte destinatário:

\section{PROF. NALI DE JESUS DE SOUZA}

Revista Análise Econômica

Av. João Pessoa, 52

CEP 90040-000 - PORTO ALEGRE (RS), BRASIL

Telefones: (051) 228-1633 - 224-6024 ramais 3440 e 3348

Fax: (051) 225-1067 


\title{
INDEXAÇÃO SALARIAL: UMA ABORDAGEM MACROECONÔMICA
}

\author{
Jo Anna Gray 1
}

\section{SINOPSE}

Este ensaio examina a importancia da indexaçăo salarial no amortecimento das flutuaçoes macroecon6́micas $\mathrm{im}$ um modelo neoclássico simples, modificado para incorporar a regidez salarial de curto prazo e a incerteza. A anflise afasta-se da literatura previa sobre a indexa-

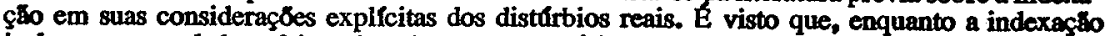
isola o setor real dos efeitos dos choques monetírios, ela pode exacerbar $\infty 8$ efeitos reais dos

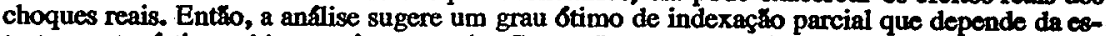
trutura estochistica subjacente à economia. Conseqüentemente, a indexactio b́tima nfo irh, em geral, isolar o setor real da variabilidade monetaria.

\section{INTRODUÇĀO}

Este artigo desenvolve uma moldura para investigar o papel da inàexação no amortecimento das flutuaçōes macroeconômicas. A característica distinta da análise e a ênfase tanto nas perturbaçōes reais quanto monetárias como fonte única de variação nos preços e na produção. 2 Concluiu-se que, enquanto â indexação isola o setor real dos cho-

\footnotetext{
1 Professora da Universidade de Rochester, Estados Unidos. A autora esta em débito com Robert Barro, Rudiger Dornbusch, Stanley Fischer e Tom Willians pelos comentários muito úteis que efetuaram em versóes preliminares deste artigo, Este trabalho foi editado originalmente pelo Journal of Monetary Economics (North Holland Publishing Company), v.2, n.2, 1978, p.221-235. A publicaçăo em língua portuguesa foi devidamente autorizada. Traduçắo de Gíacomo Balbinotto Neto, não re vista pela autora.
}

2 Os aspectos da estaijilizaçăo da indexaçăo foram reconhecidos por uma variedade de escritores, incluindo Jevons (1896), Marshall (1925) e Pigou (1933), como também Friedman (1974). Entretanto, com exceçăo de Pigou (1933, p. 295), esses escritores confinam suas discussores a estruturas que, implicitamente ou explicitamente, admitem somente disturbios monetários.

\begin{tabular}{|l|l|l|l|l|}
\hline ANÁLISE ECONÔMICA & ANO 10 & No 17 & MARÇO/92 & P.3-20 \\
\hline
\end{tabular}

Neto(Mestre em Economia - UFRGS; professor da FCEJUFRGS), nfovista pela autora. 
ques monetários, pode exacerbar os efeitos reais dos choques reais. Para uma economia sujeita a ambos os tipos de choques, este resultado questiona a recomendação usual da completa indexação como uma cura para os males da incerteza monetária. Mais precisamente, a análise sugere um grau ótimo de subjacente da economia. Entre as conclusões do artigo estão as seguintes: o incentivo para indexar está relacionado à variabilidade do nível de preços, e não à taxa média de mudança. $O$ grau ótimo de indexação é parcial, não completo. A indexação não vai, em geral, neutralizar completamente a variabilidade monetária; então as políticas que trazem aumento na incerteza monetária impõem custos inevitáveis à economia.

O objetivo do artigo $\varepsilon$ o de desenvolver, na seguncia seção, um modelo macroeconômico estocástico simples que é subsequientemente sujeito, na seção três, a vários experimentos que ilustrarn o impacto da indexação na amplitude das flutuaçōes macroeconômicas. Na seção quatro, um grau 6 timo de indexação $€$ derivado e, na seção cinco, $€$ explorado o custo do aumento da variabilidade monetária. Um sumário das conclusões e implicações do artigo está contido na seção final.

\section{MODELO}

A estrutura de tempo discreto descrito nesta seção é um modelo neoclássico simples modificado para incorporar as inflexibilidades dos salários no curto prazo e a incerteza. As inflexibilidades dos salários são produzidas por um esquema de contrato que requer o estabelecimento de uma taxa de salário-base nominal e de um parâmetro de indexação, antes que sejam recebidas informações completas sobre as variáveis econômicas relevantes às decisões da produção. A incerteza $\epsilon$ incorporada na forma de elementos estucásticos na oíerta de moeda e na função de produçãe que geram, respectivamente, choques monetários e reais para o sistema. Desde que o salário-base nominal é fixado, estes choques podem causar oscilaçôes no emprego e na produção, através de alterações na taxa de salário real (via oscilação no nível de preço) e no produto marginal do trabalho. A importância do parâmetro de indexação originase do seu papel na determinação da sensibilidade da taxa de salário real às variaçốes no nfvel de preço.3 Se o parâmetro de indexação for íxado em zero, não $€$ feito «enhum ajustamento no salário-base nominal negociado em relação à variação no nível de preços e, em conseqüência, a taxa de salário real varia inversamente com os preços. Se, por outro lado,

3 parametro de indexaçăo $(\gamma)$ é formalmente definido na seçāo quatro. Para os propósitos da

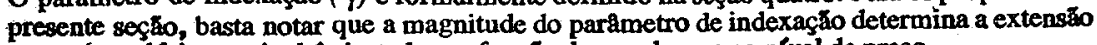
no qual o salário nominal $e$ ajustado em função das mudanças no nível de preço. 
o parâmetro de indexaçāo for fixado em um, o salário-base é completamente ajustado as alterações no poder de compra e a taxa de salário real se mantém constante face às flutuações do nível de preços. Como é demonstrado nas seções seguintes, a vantagem de uma taxa de salário real que não seja suscetf́vel às variaçōes do nf́vel de preços - e, portanto, a atratividade da indexação - depende se as variações são geradas por distúrbios reais ou monetários.

O artigo está preocupado com a distinção entre choques agregados reais e nominais. Conseqüentemente, flutuações nos preços relativos e nas quantidades são excluídas pela especificação de um modelo de um só bem. Uma vez que a indexação objetiva dar proteção contra movimentos não esperados no nível de preços, o modelo abstrai variações antecipadas e tendências, postulando distúrbios estocásticos com média zero.4 $O$ estoque de capital $\epsilon$ pressuposto ser fixo, então o produto agregado pode ser escrito como função do insumo trabalho total e de um fator de produtividade $\alpha: 5$

$$
\alpha_{t}=\alpha_{t} G\left(L_{t}\right), \quad \alpha_{t}=1+\mu_{t} .
$$

$G\left(L_{t}\right)$ e homogêneo de grau menor que 1 e $\mu_{t}$ é um termo estocástico simetricamente distribuŕdo com média zero.

A oferta nominal de moeda $€$ gerada pelo processo estocástico,

$$
M_{t}^{S}=\beta_{t} \bar{M}^{\prime} \quad \beta_{t}=1+\xi_{t},
$$

onde $\bar{M}$ E constante e $\xi_{t}$ e um termo estocástico simetricamerite distriburdo com média zero. Note-se que $\xi_{t}$ e $\mu_{t}$ não são correlacionados. A demanda por encaixes monetários nominais $\varepsilon$ assumida, por simplicidade, que tome a forma de Cambridge:

$$
M_{t}^{D}=k P_{t} Y_{t}
$$

onde $k$, a proporção desejada de dinheiro em relação à renda nomi-

4 Mudanças plenamente antecipadas no nfvel de preço săo toraadas em consideraçăo durante as negociaçб̄es do contrato e não necessitam ajuste ex-post da taxa de salário nominal em funçฐ̆o da mudança do nfvel de preço que a indexaçăo oferece. Portanto, numa economia que experimenta taxa de inflaçăo perfeitamente antecipada e constante, nfto ha razto para a

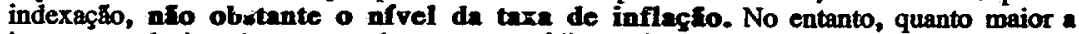
incerteza relacionada com qualquer taxa média de inflaçắ, mais fortes as razbes para indexar. Assim, o incentivo a indexar esta relacionado a variabilidade do nivel de progo (imperfeitamente antecipado), e näo d̀ taxa media de variaçăo.

5 A estrutura especificada nesta seçæ̋o pode ser vista como uma aproximaç̧o do sistema $\log$-linear desenvolvida na seçắ quatro. Em particular os termos variáveis $p$ e $\xi$ da eccilo quatro correspondem aos valores logaritmizados de $a$ e $\beta$ da presente soglto. 
nal é constante. Conseqüentemente, a análise não considera mudanças estocásticas na velocidade. Assume-se que os preços sejam ajustados instantaneamente para assegurar o equilibrio no mercado monetário. 6

$$
M_{t}^{S}=M_{t}^{D}
$$

O nfvel de emprego depende da natureza dos contratos e da produção tanto quanto das condições do mercado de trabalho. É assumido que a produção seja um processo discreto que tenha lugar uma vez em cada perfodo. Todos os contratos têm a duração de um perfodo e estabelecem uma taxa base nominal $\left(W^{*}\right)$ e um parâmetro de indexação $(\gamma)$. Contratos por qualquer perfodo $t$ são firmados no final do perfodo $t-1$, então estas duas variáveis de controle precisam ser estabelecidas com informação incompleta a respeito das outras variáveis relevantes para as decisōes de produção no período $t$. Várias complicações em potencial são evitadas, supondo-se que o salário-base seja determinado no nível que corresponda ao equilibrio no mercado de trabalho quando os valores realizados dos doîs termos de perturbação são zero. 7 Este nivel é designado por um asterisco e é refierido como sendo o equivalente certo da ta$x a$ de salário nominal. ${ }^{8} \mathrm{O}$ mercado de trabalho é descrito pelas equações (5) e (6). As funções oferta e demanda assumem formas familiares, com a demanda dependendo da razão da taxa de salário real $w$, e do fator de produtividade $\alpha: 9$

$$
\begin{array}{ll}
L_{t}^{D}=f\left(\frac{w_{t}}{\alpha_{t}}\right), & W_{t}=W_{t} / P_{t}, f_{w / a}<o, \\
L_{t}^{S}=g\left(w_{t}\right), & g_{w}>o .
\end{array}
$$

6 Usando a Lei de Walras, o mercado de bens foi eliminado do sistema. A inclusão explfcita deste mercado envolveria uma funçåo de excesso de demanda agregada da forma

$E D Y=Y_{t}^{D}-Y_{t}^{S}=H\left(M_{t}^{S / P}-M_{t}^{D / P}\right)$,

onde

$H$ C uma funçăo crescente da funçăo excesso de oferta no mercado monetário e $H(O)=0$. Sob esta especificaçăo, o equilfbrio no mercado monetário automaticamente implica o equilfbrio do mercado de bens.

7 Nh̆o há razర̌es para presumir-se que a taxa salarial negociada pelos agentes econ6́micos maximizadores de utilidade seria o equivalente certo ao especificado aqui. A preocupaçåo deste artigo, no entanto, $\varepsilon$ com o parametro de indexaç゙๋, e năo a taxa salarial, como uma variável de controle. De acordo com isso, o problema de especificar uma regra de salário que se aproxime ao comportamento maximizador é desprezfvel e o cenário de mecanismo de salário e estipulado.

8 Este conceito nfo deve ser confundido com a esperança matemática da taxa de salário nominal. desde que, em geral, os dois conceitos năo săo equivalentes.

9 A razblo de $w$, o $\alpha_{t}$ pertencem a função de demanda por trabalho como uma razh̆o, pode ser vista pela diferenciaçăo da funçto de produçẳo com respeito ao trabalbo e igualando o resultado (produto marginal do trabalho) 1 taxa de salário real. 
Então, a taxa de salário-base nominal pode ser determinada igualando a demanda e a oferta de trabalho, sujeita à restrição de que os distúrbios estejam em suas médias $\mu_{t}=\xi_{t}=O$.

Os determinantes do parâmetro de indexação são discutidos em detalhe na seção quatro. Para os objetivos atuais, pode ser notado que o valor do parâmetro deverá estar entre zero e um, inclusive.

Uma vez que os contratos tenham sido negociados, os valores de todos os termos estocásticos previamente desconhecidos associados com o perfodo $t$ são realizados e as decisões de produção são tomadas. Neste ponto o nível de emprego torna-se completamente determinado pela demanda. É assumido que os trabalhadores ofertem qualquer quantidade de trabalho demandado pelos empregadores à taxa de salário negociada. Com efeito, uma vez que a taxa de salário esteia determinada, a oferta dẹ. trabalho torna-se perfeitamente elástica e a eq. (6) E afastada do sistema. ${ }^{10}$

\section{RESPOSTÁ DOS SISTEMAS AOS CHOQUES}

Nós voltamos agora a examinar alguns casos especiais indicados para ilustrar o impacto da indexação na amplitude das flutuaçōes macroeconômicas. É mostrado que os efeitos da indexação dependem crucialmente de se as variações no nível de preços são geradas por distúr bios reais ou mưnetários. 11 Enquanto indexar erita a transmissão dos choques monetários para o setor real, também exacerba os efeitos reais dos distúrbios reais. Nós começamos por postular uma economia sujeita somente a choques monetários. O comportamento do sistema E explorado sob dois pressupostos de indexação alternativas: nenhuma indexação e indexação incompleta. $\mathbf{O}$ mesmo conjunto de experimentos $\varepsilon$ subseqüentemente executado em uma economia sujeita a distúrbios reais.

\subsection{Choques Monetários}

iNesta seção somente os choques monetários são considerados ao sistema. A possibilidade de distúrbios reais é eliminada reduzindo a zero o termo estocástico na função de produção, deixando o produť agrega-

10 As conclusర్es deste artigo são robustas com respeito a variaçరes nostex pressupostos. Os mesmos resultados qualitativos são obtidos se, por exemplo, o emprego for determinado por uma regra "short end" (emprego sendo o menor dos montantes demandados e ofertados). $O$ pressuposto sobre a qual a analise depende crucialmente é que o que såo negociados sło os níveis de salário, antes que os nf́veis de emprego ou renda.

11 Fischer (1975) chega a conclusðes similares usando uma estrutura que está de foordo com o presente artigo. Para uma visão alternativa da natureza dos resultados e da determinaçðo do produto e emprego e, conseqüentemente, o impacto de indexar $\mathrm{em}$ flutuaģes macroeconómicas, veja Barro (1975). 
do exclusivamente como função do insumo trabalho total,12

$$
Y=G(L) \text {. }
$$

Da mesma forma, a demanda por trabalho neste caso depende unicamente da taxa de salário real:

$$
L^{D}=f(w) \text {. }
$$

A resposta do sistema a um aumento inesperado na oferta de moeda pode ser analisada usando a fig. 1, que dá uma interpretação geometrica do impacto do choque monetário sobre o setor real. No quadrante 1 é representada a dependência da taxa de salário real sobre o termo de perturbação $\xi$. A natureza da dependência $e$ condicionada ao pressuposto de indexação empregada; no caso de uma economia indexada, a curva $€$ perfeitamente elástica, enquanto que, para um sistema não indexado, é negativamente inclinada. Estes resultados podem ser obtidos das equacóes (2 e (3) e do pressuposto de equilíbrio contínuo do mercado monetário. Fazendo a oferta nominal de moeda igual à demanda temos

$$
\beta \bar{M}=k P Y, \quad \beta=I+\xi .
$$

Observando a definição da taxa de salário real $(w=W / P)$ e a dependência exclusiva do produto sobre o salário real (via nível de emprego), a eq. (7) pode ser reescrita de uma forma mais reveladora.

$$
(I+\xi) \cdot \bar{M}=k(W / w) . G[f(w)], \quad G_{w}<0 .
$$

$\grave{A}$ medida que $\xi$ aumenta, a oferta nominal de moeda se expande. $O$ equilíbrio contínuo no mencado monetário exige um aumento correspondente na demanda por encaixes nominais, como a dada pelo lado direito da eq. (8). Isto pode ser obtido por uma queda na taxa de salário real, por um aumento na taxa de salário nominal, ou ambos. É neste ponto que torna-se importante a distinçāo entre sistemas indexados e não-indexados. Em uma economia não-indexada, o parâmetro de indexação $€$ fixado em zero e nenhum ajustamento na taxa de salário nominal é feito para variações no nivel de preços. Entāo, para um sistema nāo-indexado a taxa de salário nominal (W) da eq. (8) E um parâmetro fixo e a taxa de salário real $(w)$ precisa cair em resposta ao choque monetário

12 Os subscritos referentes ao tempo sāo omitidos aqui e nas seçбes seguintes: 
postulado. Pc tanto, há uma relação inversa entre $w$ e $\xi$ para $\gamma=O$, como e mostrado na fig. 1. O intercepto desta curva corresponde ao equivalente certo da taxa de salário real. Numa economia indexada, por outro lado, o parâmetro de indexação é fixado na unidade e a taxa de salário nominal é completamente ajustada a variações no nfvel de preços. Então, a taxa de salário real é efetivamente fixada em seu equivalente certo, significando uma curva perfeitamente elástica retratada para $\gamma^{\prime}=$ 7 .

Quadrante II Quadrante I

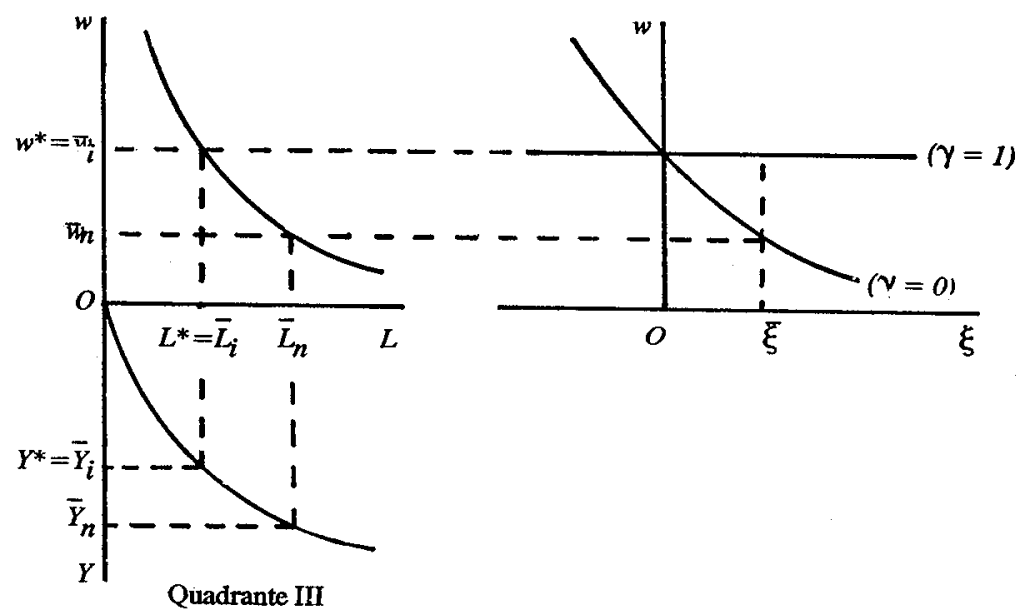

Figura 1

No segundo quadrante a demanda por trabalho é mostrada como uma função da taxa de salário real (eq. 5) e, no terceiro quadrante, a função de produção (eq. 1) é mostrada.

Correspondendo a $\xi=O$, e denotados por asteriscos, estão os equivalentes certos de taxa de salário real, emprego e produto, que caracterizam a economia em sua média. É prontamente verificado que, para uma economia não-indexada, um aumento inesperado da oferta de moda leva a niveis de emprego e produtos excessivos em seus equivalentes certos. Considere, por exemplo, os efeitos de um choque monetário positivo como $\xi$. O choque resulta em uma taxa de salário real $\bar{w}_{n}$ que $\varepsilon$ menor do que o equivalente certo do salário real $w^{*}$. Isto, por sua vez, 
induz a nfveis e emprego $\left(\bar{L}_{n}\right)$ e produto $\left(\bar{Y}_{n}\right)$ que excedem aos seus equivalentes certos $\left(L^{*}\right.$ e $\left.Y^{*}\right)$. Similarmente, a fig. 1 pode ser usada para mostrar que reduçōes inesperadas na oferta de moeda resultam em nifveis de produto e emprego menores do que $L^{*}$ e $Y^{*}$. Portanto, distúrbios monetários realmente têm efeitos reais numa economia não-indexada.

Numa economia indexada, a taxa de salário real é fixada efetivamente em seu equivalente certo e, conseqüentemente, distúrbios monetários não têm efeitos reais. Usando novamente a fig. 1 para mostrar os efeitos de um choque positivo, é evidente que a taxa de salário real $\left(\bar{w}_{i}\right)$, emprego $\left(L_{i}\right)$, e produto $\left(\bar{Y}_{i}\right)$ uma economia indexada são todas iguais aos seus equivalentes certos.

Claramente, então, o setor real é isolado dos efeitos dos choques monetários em uma economia completamente indexada. Falhas na indexação, por outro lado, causam transmissão dos distúrbios monetários para o setor real.

\subsection{Choques Reais}

Passaremos agora ao caso de uma economia sujeita somente a distúrbios reais. Para tal economia, a eq. (1) em sua forma estocástica é a função de produção relevante. Os choques monetários são exclứdos pela redução a zero dos seus termos de perturbação na oferta de moeda, desse modo fixando os encaixes nominais em $\bar{M}$,

$$
M^{S}=\bar{M}
$$

A resposta do sistema aos choques reais pode ser demonstrada usando o conjunto de diagramas similares aos da subseção anterior. No primeiro quadrante da fig. 2 , está representada a dependência da taxa de salário real ao termo de perturbação real $\mu$. Novamente, a natureza da relação é determinada pela suposição da indexação empregada; no caso de uma economia indexada, a curva é perfeitamente elástica, enquanto que, em um sistema não-indexado, é positivamente inclinada. Estes resultados são obtidos, como na seção anterior, das consições de equilifbrio para o mercado monetário.

$$
\bar{M}=k \cdot(W / w) \cdot(I+\mu) \cdot G\{f[\omega /(I+\mu)]\}
$$

onde

$$
G_{w / \alpha}=G_{w /(1+\mu)}<0 .
$$

Com a oferta nominal de-moeda fixada, um aumento em $\mu$ precisa 
ser compensado por um aumento na taxa de salário real, uma redução na taxa de salário nominal, ou ambos. Em uma economia não-indexada a taxa de salário nominal é fixa, de forma que a carga dos ajustes recai sobre a taxa de salário real, que aumenta em resposta a aumentos em $\mu$. Isto resulta numa relação direta entre $w$ e $\mu$, obtida para $\gamma=O$ na fig. 2 . Numa economia indexada, a taxa de salário real é fixa em seu equivalente certo, implicando uma curva perfeitamente elástica para $\gamma=1$.

No segundo quadrante, a demanda por trabalho e novamente representada como uma função da taxa de salário real. Nesta subseção, entretanto, a demanda por trabalho depende do fator de produtividade, $\alpha=$ $l+\mu$, tanto quanto da taxa de salário real. Então, os aumentos na produtividade associados com aumentos em $\mu$ causam mudanças externas nesta curva. Da mesma forma, a posição da função de produção mostrada no terceiro quadrante é dependente de $\mu$. Maiores valores de $\mu$ correspondem a maiores nfveis de produto, a qualquer nfvel de emprego e, portanto, a mudanças para cima na curva de produçāo.

Usando a fig. 2, pode ser mostrado que os efeitos no produto e no emprago de um choque real são maiores numa economia indexada do que numa economia não-indexada. Considere, por exemplo, um choque real positivo como $\not$. Em um sistema não-indexado o choque resulta em uma taxa de salário $\bar{w}_{n}$ que excede ao equivalente certo $w^{*}$. Ao mesmo teinpo, a curva de demanda por trabalho move-se para fora, e a função de produção para baixo, relativo às curvas associadas com $\mu=O$. É facilmente verificado que o deslocamento vertical da curva de demanda por trabałho em $L^{*}$ é igual ao aumento da taxa de salário real induzida pelo distúrbio. 13 Conseqüentemente, o aumento na taxa de sálário $\varepsilon$ exatamente compensado por maior produtividade no trahalho, e os empregadores não têm incentivo para alterar o nf́vel de emprego inicial de $L^{*} .{ }^{14} \mathrm{Em}$ tal $\mathrm{n}\left(\mathrm{vel}\right.$ de emprego, entretanto, o produto $\left(F_{n}\right)$ excede ao

13 A mudança na taxa de salário real (relativo a $w^{*}$ ) induzida por $\mathbb{F}^{*}$ mantendo o emprego constante em $L^{*}$ e dada por

$\mathrm{d} \log \mathrm{W}=\mathrm{dlog} \mathrm{W}-\mathrm{dlog} \mathrm{P}=\mathrm{d} \log \mathrm{Y}=\overline{\boldsymbol{\mu}}$

A mudança correopondente no produto marginal do trabalho e

$\operatorname{dlogMPL}=\operatorname{dlog}[\alpha(G / L)]=p$

em um dado nível de emprego $L^{*}$, entāo, dlogw $=$ dlog $M P L$.

14 Isto também pode ser visto na eq. (5), que especifica a demanda de trabalho como uma funçảo da razăo entre a taxa real de salário e o fator de produtividade $\alpha$. Em uma economia năo-indexada, se o emprego $\&$ mantido constante em $L^{*}$,

$d \log w=-d \log P=d \log Y=d \log \alpha$,

então a razão w/ $\alpha$ e uma constante. Conseqũentemente, choques reais năo têm efeitos no nivel de emprego sum sistema nåo-indexado. 
seu equivalente certo $\left(Y^{*}\right)$ devido ao aumento de produtividade associado com $\boldsymbol{\pi}$. Similarmente a fig. 2 pode ser usada, para mostrar que um decréscimo não antecipado da produtividade resulta num nível de produto que $\varepsilon$ insuficiente para $Y^{*}$, enquanto o emprego $\varepsilon$ mantido em $L^{*}$. Conseqüentemente, numa economia não-indexada, o impacto de um choque real sobre o produto real é limitado pela resposta da taxa de salário real, o qual se ajusta para inanter o emprego fixo em $L^{*}$.

Numa economia indexada, a taxa de salário real é efetivamente fixada em $w^{*} e$, como conseqüência, não exerce influência moderadora sobre a resposta do produto demonstrado no caso não-indexado. Usando novamente a fig. 2 para traçar os efeitos de um choque real positivo, $\varepsilon$ evidente que o emprego $\left(\vec{L}_{i}\right)$ excede $L^{*}$. Portanto, o nivel de produto num sistema indexado excede ambos seus equivalentes certos $\left(Y^{*}\right)$ e o nivel alcançado no sistema não-indexado $\left(\bar{Y}_{n}\right)$. Claramente, o efeito real dos distúrbios reais são mais pronunciados numa economia indexada do que em uma economia não-indexada.

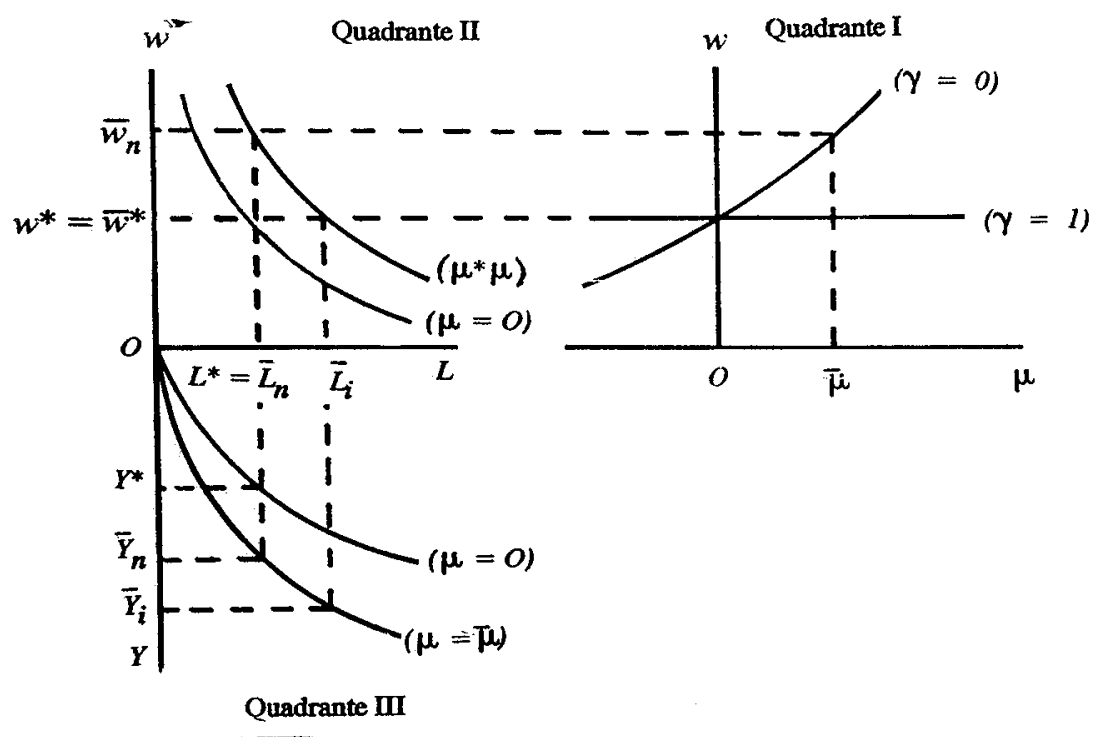

Förra2

A análise da seção três demonstrou as diferenças nas respostas da economia indexada e não-indexada aos choques reais e monetários. As conclusões da seção são diretas: enquanto indexar $\widehat{e}$ um meio efetivo de isolar o setor real dos choques monetários, ela exacerba os efeitos reais 
dos distúrbios reais Neste ponto, parece atraente concluir que, nunca economia sujeita tanto a choques monetários como reais, precisa existir um grau de indexação entre zero e um que seja de alguma forma ótimo. Até agora, entretanto, nenhum critério foi estabelecido para a escolha racional de um grau ótimo de indexação. Em particular, a extensão, em que $\varepsilon$ desejável moderar as respostas reais aos choques reais, at $\varepsilon$ agora não foi discutida. Estas questões estão contidas na seção quatro, na qual é proposto um critério de bem-estar baseado em desvios do produto real de um nível "desejado" de produto e derivado um grau ótimo de indexação.

\section{O GRAU ÓTIMO DE INDEXAÇĀo}

Nesta parte, a estrutura formal da seção dois é expandida para incorporar os elementos remanescentes necessários a uma discussão do grau ótimo de indexação. Para facilidade de cálculo, as eqs. de (1) a (6) são reespecificadas na forma de logaritmo linear:

$$
\begin{aligned}
& \log Y=\delta \log L+\mu, \\
& \log M^{S}=\log \bar{M}+\xi, \\
& \log M^{D}=\log k+\log P+\log Y, \\
& \log M^{S}=\log M^{D}, \\
& \log L^{D}=-\eta(\log w-\mu)+\eta \log \delta, \quad \eta=1 /(1-\delta),
\end{aligned}
$$

$\log L^{S}=\omega \log w+\eta \log \delta$.

Os termos estocásticos $\mu$ e $\xi$ são distribufdos simetricamente com média zero. As elasticidades da oferta e da demanda por trabalho são dadas, por $\omega$ e $\eta$, respectivamente enquanto $\delta$ e a elasticidade do produto

15 A função de demanda por trabalho e derivada pela diferenciação da função de produção.

$Y=e^{\mu}{ }_{L}^{\delta}$

com respeito ao trabalho e igualando o resultado (produto marginal do trabalho) a taxa real dé salários. Isto dá uma funçăo de demanda na forma

$$
\begin{aligned}
& L=\left(w / \delta e^{\mu}\right)^{1 / \delta-I}, \\
& q u e, \text { em forma de log, é a eq. ( }\left(^{\prime \prime}\right) .
\end{aligned}
$$


real com respeito ao insumo trabalho. $O$ termo constante na funçāo de oferta de trabalho, $\eta \log \delta$, escolhido de maneira que $\log w^{*}$ (o equivalente certo do logw) seja igual a zero.

Pressupõe-se que os tomadores de decisões sejam neutros ao risco e racionais, e avaliem corretamente a estrutura estocástica da economia e, com base nesta determinação, atuem para maximizar o bem-estar social. É assumido que o critério de bem-estar adquira a forma de função de perda, $Z$, que $E$ expressa em termos de desvio do logaritmo do produto corrente $(\log Y)$ e do logaritmo de um nível "desejado" de produto $\left(\log Y_{0}\right):$

$$
Z=E\left[(\log Y-\log Y \circ)^{2}\right] .
$$

O produto desejado $\epsilon$ definido como sendo aquele nfvel de produto que predominaria em uma versão isenta de fricções da economia pressuposta neste artigo. Nesta economia, o valor do logaritmo do produto seria dado por,

$$
\log Y_{\mathrm{o}}=\delta \log L_{\mathrm{o}}+\mu
$$

onde $L_{0}$ e o nfvel de emprego correspondente à intersecção da oferta de trabalho e da curva de demanda pelas equaçóes (5) e (6). A motivação para definir o produto desejado desta maneira origina-se na ineficiência social implícita pelos pontos que estâo fora das curvas de oferta e demanda de mercado. Similarmente, a forma particular que a funçāo de perda assume na eq. (10) é delineada para aproximar a perda no excedente do consumidor associada com desvios do produto ótimo.

No apêndice, as expressōes seguintes sāo desenvolvidas para logaritmos do produto real e ótimo:

$$
\begin{gathered}
\log Y=\delta \eta\left[\frac{(1-\gamma) \xi+\gamma \mu}{1+\delta \eta(1-\gamma)}\right]+\mu+\delta \eta \log \delta, \\
\left.\log Y_{\circ}=\delta \eta \mu / \omega /(\omega+\eta)\right]+\mu+\delta \eta \log \delta .
\end{gathered}
$$

Subtraindo (13) de (12), elevando ao quadrado os resultados e levando em conta as expectativas, resulta na seguinte expressão para a função de perda,

$$
Z=\delta^{2} \eta^{2}\left\{V_{\mu}\left[\frac{\gamma}{I+\delta \eta(I-\gamma)}-\frac{\omega}{\omega+\eta}\right]^{2}+V \xi\left[\frac{(I-\gamma)}{1+\delta \eta(I-\gamma)}\right]^{2}\right\}_{(14)}
$$


Aqui $V_{\mu}$ e $V \xi$ são as variâncias dos termos de perturbação $\mu$ e $\xi$, respectivamente.

Desde que o bem-estar ê maximizado quando o valor da função de perda, $Z$, é minimizada, o grau ótimo de indexação (denotado por $\gamma_{0}$ ) pode ser obtido por condições de primeira ordem para um mínimo. Diferenciando a eq. (14) em relação a $\gamma$, igualando o resultado a zero e isolando $\gamma_{0}$, temos

$$
\gamma_{0}=\theta+(1-\theta)[\omega=/(1+\omega)]
$$

onde

$$
\theta=\frac{V_{\xi}}{\left[\frac{\eta^{2}(I+\omega)}{\omega+\eta}\right] V_{\mu}+V_{\xi}}
$$

O exame dos termos que compōem a eq. (15) revelam que o grau ótimo de indexação, $\gamma_{o}$, e uma média ponderada dos ótimos correspondentes aos casos extremos $V_{\mu}=O$ e $V_{\xi}=O$. Igualando $V_{\mu}$ a zero, resulta $\gamma_{\circ}$ igual a um. Este $\varepsilon$ o caso discutido na seção 3.1 de uma economia sujeita somente a choques monetários. A racionalidade por trás de uma política de indexação plena em uma economia como esta é clara.

Quando, por outro lado, $V_{\xi}$ é igualado a zero (o caso de uma economia sujeita somente a choques reais), $\gamma_{0}$ toma o valor $\omega /(1+\omega)$, que E não negativo e menor que um. A racionalidade por trás deste resultado apoia-se na observaçāo de que é desejada alguma resposta do setor real aos choques reais. Voltando brevemente à estrutura da seção três, considere o caso de um choque real positivo com $\bar{\mu}$ (fig. 3). Os niveis de produto e emprego desejados, $Y_{\circ}$ e $L_{0}$, excedem àqueles obtidos em economias não-indexadas, mas são insuficientes em economias completamente indexadas. Claramente, os níveis desejados podem ser obtidos através de algum grau de indexaşão menor que um mas maior que zero. Dentro da estrutura especiffica da presente seção, este grau ótimo de indexação é independente da elasticidade da demanda por trabalho $(\eta)$, mas é diretamente relacionado à elasticidade da oferta de trabalho $(\omega)$. A manipulação das inclinações das curvas obtidas na fig. 3 fornece as bases intuitivas destes resultados.

Para o caso geral no qual as variâncias de ambos os termos de perturbação não são zero, $\gamma_{0}$ situa-se entre $l$ e $\omega(l+\omega)$. A dependência de $\gamma_{0}$ sobre magnitudes relativas dos dois tipos de distúrbios é refletida nos pesos $\theta$ e $(l-\theta)$. Se o tamanho relativo dos distúrbios monetários $V_{\xi}$ aumenta, $\theta$ aumenta e $\gamma_{0}$ se aproxima da unidade. In- 
versamente, à medida que o tamanho relativo dos choques reais $\left(V_{\mu}\right)$ aumenta, $(l-\theta)$ aumenta e $\gamma_{0}$ se aproxima de $\omega(1+\omega)$. Muito menos óbvia é a dependência do grau ótimo de indexação sobre elasticidades da oferta e da demanda por trabalho. Pode ser mostrado, entretanto, que $\gamma_{0}$ e positivamente relacionado a $\omega$ e negativamente relacionado a $\eta$.

Quadrante II

Quadrante I

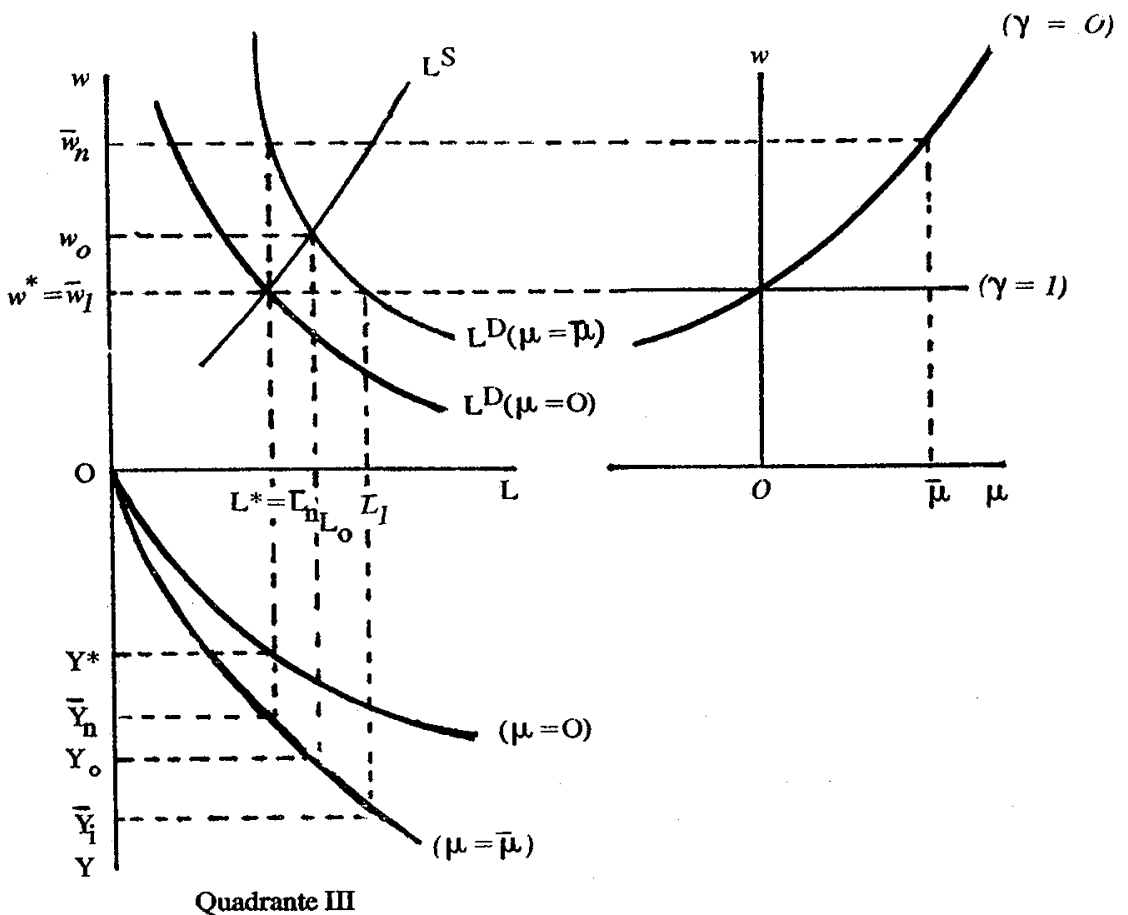

Figura 3

\section{OS CUSTOS DO AUMENTO DA VARIABILIDADE MO- NETÁRIA}

Uma implicação importante dos modelọs que incorporam distúrbios monetários, mas que negligenciam choques reais $\varepsilon$ a seguinte: numa economia livremente ajustada, otimamente indexada, um aumento na variabilidade monetária não impōe custos. Nesta seção, nós mostramos 
que, para o caso mais geral de um sistema sujeito a ambos os distúrbios, reais e monetários, a proposição acima não ê válida.

Formalmente, os custos de um aumento na variabilidade monetária podem ser mostrados diferenciando-se a função de perda dada pela eq. (14) em relação a $V_{\xi}$, permitindo um ajustamento ótimo do parâmetro de indexação ao aumento da incerteza monetária,

$$
\begin{aligned}
& d Z J d V \xi=\partial Z / \partial V \xi+\left(\partial Z / \partial \gamma_{0}\right)\left(d \gamma_{0} d d V_{\xi}\right) \\
& =\delta^{2} \eta^{2}\left[\frac{\left(I-\gamma_{0}\right)}{1+\eta \delta\left(I-\gamma_{0}\right)}\right]^{2} \\
& >\quad 0 .
\end{aligned}
$$

Como a eq. (16) demonstra, um aumento na variabilidade monetária definitivamente impõe custos, mesmo numa economia livremente ajustada e otimamente indexada.

Um argumento menos formal provém das conclusões da última seção. Já foi mencionado que, num sistema sujeito a choques tanto reais como monetários, o parâmetro de indexação não seră fixado na unidade. $O$ isolamento pleno do setor real aos choques monctários, então, não $\varepsilon$ alcançado. À medida que a variabilidade monetária aumenta, o parâmetro de indexação aumentará parcialmente, mas não completamente, compensando o aumento na incerteza monetária. A natureza parcial desta compensação origina-se do aumento do custo dos choques reais induzidos por aumentos em $\%$. O sistema é deixado, então, com um aumento lfquido nos custos.

\section{COMENTÁRIOS FINAIS}

Neste artigo, nós demonstramos a proposição de que, em uma economia otimamente indexada, o setor real não será, em geral, completamente isolado dos distúrbios monetários. Isto é porque o grau b́timo de indexação em tal economia é menor que um, se os choques reais formam parte da estruiura estocástica do sistema. Estas condusões baseiam-se numa distinção eritre as flutuações no nfvel de preços causadas por choques monetários e aqueles causados por choques reais. A análise implica que as políticas governamentais que trazem aumento na incerteza monetária impōem custos inevitáveis sobre a economia. Indexar não pode, em geral, neutralizar completamente a variabilidade monetária, isto parece, portanto, ser um substituto inadequado para um comportamento inteligente por parte da autoridade monetária. 


\section{APÊNDKCE}

\section{PRODUTTO CORRENTE}

O produto comente e dado pela eq. (1") do texto, como função do insumo trabalho, $L$, e do fator de produtividade, $\mu$. $O$ insumo trabalho corrente, por sua vez, e completamente determinado pela demanda e, portanto, dado pela eq. (5") do texto. Substituindo (5") em (1") temos:

$$
\begin{aligned}
\log Y & =-\delta \eta(\log w-\mu)+\mu+\delta \eta \log \delta . \\
\log w & =\log W-\log P \\
& =\log W^{*}+\gamma\left(\log P-\log P^{*}-\log P, \quad \gamma=\frac{\log W-\log W^{*}}{\log P-\log P^{*}}(\mathrm{~A} 2)\right.
\end{aligned}
$$

(Lembrar que os asteriscos denotam equivalentes certos, ou vaiores correspondentes a $\mu=\xi=0$ ). Fazendo $\log w^{*}=0$, resulta em $\log W^{*}=\log P^{*}$, então (A2) pode ser escrita como

$$
\log w=(\gamma-1)\left(\log P-\log P^{*}\right) .
$$

Substituindo (A3) em (A1), temos

$$
\log Y=-\delta \eta(\gamma-1)\left(\log P-\log P^{*}\right)-\mu J+\mu+\delta \eta \log \delta
$$

Igualando as equações (2") e (3") do texto e resolvendo para $\log P$, resulta:

$$
\log P=\log \bar{M}+\xi-\log k-\log \bar{Y} .
$$

Fazendo $\xi=\mu=O$, temos

$$
\log P^{*}=\log \bar{M}-\log k-\log Y^{*} \text {. }
$$

Por sua vez, $\log Y^{*} \leq$ dado por

$$
\log Y^{*}=\delta \log L^{*}=\delta \eta \log \delta .
$$

(Lembrar que $\log w^{*}=0$ ). Substituindo este resultado em $(\mathrm{A6})$ e subtraindo (A6) de (A5), temos

$$
\log P-\log P^{*}=\xi-\log Y+\delta \eta \log \delta .
$$


Substituindo (A8) em (A4) e resolvendo log Y resulta;

$$
\log Y=\delta \eta\left[\frac{(l-\gamma) \xi+\gamma \mu}{I+\delta \eta(I-\gamma)}\right]+\mu+\delta \eta \log \delta,
$$

que é a eq. (12) do texto.

\section{PRODUTO DESEJADO}

O produto desejado $€$ dado por

$$
\log Y_{0}=\delta \log L_{0}+\mu
$$

onde o nivel desejado do insumo trabalho, $L_{\circ}$, é determinado tanto pelas condiçōes de oferta e demanda no mercado de trabalho. Conseqüentemente, $\log L_{\circ}$ é encontrado igualando as equaçōes (5") e (6") do texto, resolvendo para $\log w$, e subtraindo a expressão rèsultante de novo em (6")

$$
\log L_{0}=\mu(\omega \eta /(\omega+\eta))+\eta \log \delta .
$$

Substituindo a eq. (A10) em (A9) resulta:

$\log Y_{0}=\delta \eta \mu(\omega /(\omega+\eta))+\mu+\delta \eta \log \delta$,

que é a eq. (13) do texto.

\section{BIBLIOGRAFIA}

BARRO, R.J.Indexation in a Rational Expectations Mociel. New York: Univensity of Rochester, 1975. (Unpublieshed manuscript).

FISCHER, S. Wage Indexation and Macro-Economic Stability.Paper presented at the Carnegic-Rogester Conference on Public Policy, 1975.

FISCHER, I. The Purchasing Power of Money. New York, MacMillan, 1922.

FRIEDMAN, M. Monetary Correction. In: GIERSH et alii. Eratse on Inflation and Ideration. Washington, DC: American Enterprise Institute, 1974.

JEVONS. W.S. Money and the Mechanisa of Exchange. New York: D. Appleton, 1896. MARSHALL, A. Remedies of Fluctuations of General Prices, In: PIGOU, A.C.(ed.) Momories of A. Marshall. London: MacMillan, 1925.

PIGOU, A.C. Theory of Unemployment. London: MacMillan, 1933. 


\section{ABSTRACT \\ WAGE INDEXATION: A MACROECONOMIC APPROACH}

This essay examines the role of wage indexation in dampening macroeconomic fluctuations in a simple neoclassical model modified to incorporate short-term wage rigities and uncertaintv. The analysis departs from most of the previous literature on indexing in its explicit consideration of real disturbances. It is found that while indexing insulates the real sector from the effects of monetary shocks, it may exacerbate the real effects of real schocks. Thus the analysis suggests an optimal degreee of partial indexation that depends on the underlying stochastic structure of the economy. Consequently, optimal indexing will not, in general, insulate the real sector from monetary variability. 